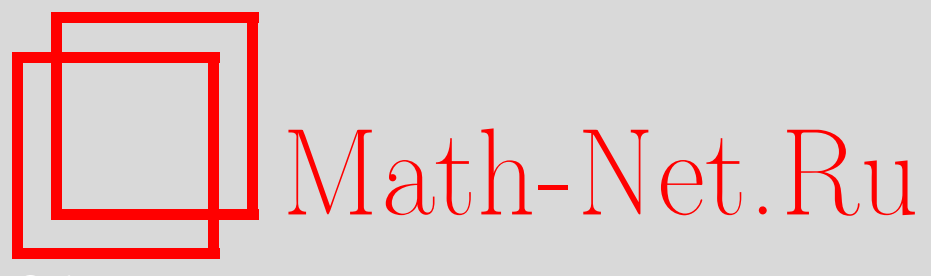

А. М. Себельдин, Д. С. Чистяков, Определяемость абелевых групп центром их кольца эндоморфизмов, $M a$ тем. заметки, 2008, том 84, выпуск 6, 952-954

DOI: https://doi.org/10.4213/mzm6569

Использование Общероссийского математического портала Math-Net.Ru подразумевает, что вы прочитали и согласны с пользовательским соглашением http://www . mathnet.ru/rus/agreement

Параметры загрузки:

IP: 54.237 .206 .68

26 апреля 2023 г., 15:25:23

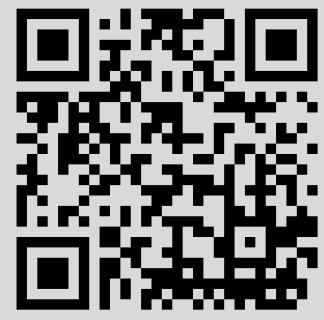




\section{Определяемость абелевых групп центром их кольца эндоморфизмов}

\section{А. М. Себельдин, Д. С. Чистяков}

Будем говорить, что абелева группа $A \in \mathbf{X}$ определяется иентром своего кольца эндоморфизмов $E(A)$ в классе абелевых групп $\mathbf{X}$, если всякий раз из изоморфизма $C(E(A)) \cong C(E(B))$, где $B \in \mathbf{X}$, следует изоморфизм $A \cong B$. Подкласс класса $\mathbf{X}$ групп, определяющихся центром своего кольца эндоморфизмов в классе абелевых групп $\mathbf{X}$, будем обозначать через $\mathbf{X}(\mathrm{cen})$. Если $\mathbf{X}(\mathrm{cen})$ содержится в нулевом классе (т.е. либо $\mathbf{X}($ cen $)=\{0\}$, либо $\mathbf{X}($ cen $)=\varnothing)$, то класс $\mathbf{X}$ будем называть $N C$-классом. То есть, если некоторый класс $\mathbf{X}$ абелевых групп является $N C$-классом, то это означает, что для любой группы $G \in \mathbf{X}$ найдется не изоморфная ей группа $H \in \mathbf{X}$ такая, что $C(E(G)) \cong C(E(H))$. Класс $\mathbf{X}$ назовем $A$-классом, если с каждой группой $A \in \mathbf{X}$ он содержит и прямую сумму ее копий $A_{\alpha}=\bigoplus_{\alpha} A$ для любого кардинала $\alpha$. Класс $\mathbf{X}$ назовем $A B$-классом, если он не является $A$-классом, но замкнут относительно конечных прямых сумм, т.е. $A, B \in \mathbf{X}$ влечет $A \oplus B \in \mathbf{X}$. Положим $P(A)=\{p \in P \mid p A=A\}$, где $P$ - множество всех простых чисел.

Через $\mathbf{A}, \mathbf{F}, \mathbf{L}, \mathbf{F}_{\mathrm{cd}}, \mathbf{F}_{1}, \mathbf{F}_{n}, \mathbf{S}$ обозначим соответственно классы всех абелевых групп, абелевых групп без кручения, периодических абелевых групп, вполне разложимых абелевых групп без кручения, абелевых групп без кручения ранга 1 , вполне разложимых абелевых групп без кручения фиксированного конечного ранга $n$, сепарабельных абелевых групп без кручения. Остальные обозначения стандартны и могут быть найдены в [1], [2] или вводятся по ходу изложения.

ПредЛОЖенИе 1. Классы $\mathbf{A}, \mathbf{F}, \mathbf{L}, \mathbf{F}_{\mathrm{cd}}, \mathbf{S}$ являются $N C$-классами.

ДокАзАтЕльство предложения следует из того факта, что любой $A$-класс является $N C$-классом. Действительно, поскольку $C(E(A)) \cong C\left(E\left(A_{\alpha}\right)\right)$, то в качестве группы $B$ достаточно взять группу $A_{\alpha}$ с $\alpha=2^{r(A)}$.

Теорема 2. Любой $A B$-подкласс $\mathbf{L}(A B)$ класса $\mathbf{L}$ является $N C$-классом.

ДокАЗАТЕЛЬСТво. Пусть

$$
A=\bigoplus_{p \in S(A)} A_{p}, \quad S(A)=\left\{p \in P \mid A_{p} \neq 0\right\},
$$

тогда $C(E(A)) \cong \prod_{p \in S(A)} C\left(E\left(A_{p}\right)\right)$. Далее рассматриваем примарный случай. Группу $H_{p}$ построим следующим образом. Если $A_{p}$ - ограниченная группа, $A_{p}=A_{g} \oplus\langle g\rangle$, где $o(g)=p^{k}$ - максимальный среди всех порядков, то $H_{p}=\langle g\rangle$, если $A_{p} \neq 0$, и $H_{p}=\langle g\rangle \oplus\langle g\rangle$, если $A_{p}=0$. Пусть $A_{p}=B_{p} \oplus D_{p}$ - нередуцированная группа, где $D_{p}$ - делимая часть группы $A_{p}$. В этом случае полагаем

$$
H_{p}=B_{p} \oplus \bigoplus_{i=1}^{n+1} Z\left(p^{\infty}\right),
$$

если $r\left(D_{p}\right)=n<\infty$, и

$$
H_{p}=B_{p} \oplus Z\left(p^{\infty}\right)
$$

если $r\left(D_{p}\right)=\infty$. Если $A_{p}$ - неограниченная редуцированная группа, то

$$
A_{p}=\left\langle a_{1}\right\rangle \oplus \cdots \oplus\left\langle a_{k}\right\rangle \oplus A^{\prime}, \quad A^{\prime}=\left\langle a_{k+1}\right\rangle \oplus A^{\prime \prime}, \quad o\left(a_{k}\right)=p^{n(k)},
$$

где $1<n(1)<\cdots<n(k)<\cdots, k \in \mathbb{N}$. В качестве $H_{p}$ берем группу

$$
H_{p}=\left\langle a_{2}\right\rangle \oplus \cdots \oplus\left\langle a_{k}\right\rangle \oplus A^{\prime} .
$$

(C) А. М. Севельдин, Д. С. Чистяков, 2008 
Лемма 3. Пусть $G$ и $H$ - абелевы группы без кручения ранга $1, \tau(G) \leqslant \tau(H)$. Тогда $C(E(G \oplus H)) \cong E(G)$.

ДокАЗАТЕЛЬСтво. Пусть $E(G) \cong R_{1}, E(H) \cong R_{2}$, где $R_{1}, R_{2}$ - подкольца поля рациональных чисел. Если $\tau(G)=\tau(H)$, то можно считать, что $R_{1}=R_{2}=R$, поэтому $C(E(G \oplus H)) \cong R$. Пусть $\tau(G)<\tau(H)$. Выберем такие ненулевые элементы $g \in G, h \in H$, что $\chi(g)<\chi(h)$. Существует единственный $\varphi \in \operatorname{Hom}(G, H)$, для которого $\varphi(g)=h$. Так как $\operatorname{Hom}(H, G)=0$, то для любого $\eta \in C(E(G \oplus H))$ найдется $r_{2} \in R_{2}$ такой, что $\eta(h)=r_{2} h$. Тогда

$$
s \eta(h)=s r_{2} h=s r_{2} \varphi(g)=\varphi\left(s r_{2} g\right)=s \eta \varphi(g)=s \varphi \eta(g)=s \varphi(\eta(g))
$$

для некоторого $s \in \mathbb{Z}$. Из инъективности $\varphi$ следует, что $\eta(g)=r_{2} g$ и $r_{2} \in R_{1}$. Откуда $C(E(G \oplus H)) \cong R_{1}$.

Лемма 4. Пусть $G$ - сепарабельная абелева группа без кручения, $H$ - абелева группа без кручения ранга 1 и тип группы $H$ больше типа любого прямого слагаемого ранга 1 группь $G$. Тогда чентр кольца $E(G \oplus H)$ изоморфен подкольцу $E$ кольца $E(H)$, причем $P(E,+)=P(G)$.

Доказательство. Выберем такие ненулевые элементы $g \in G, h \in H$, что $\chi(g)<$ $\chi(h)$. Рассмотрим прямое слагаемое $A$ группы $G$ конечного ранга $n$, содержащее элемент $g \in A=A_{1} \oplus \cdots \oplus A_{n}$, где все $A_{i} \neq 0$. Ясно, что существует $\varphi \in \operatorname{Hom}(A, H)$, переводящий $g$ в $h$, который можно рассматривать как эндоморфизм группы $G \oplus H$. Из перестановочности его ограничения $\varphi_{i}$ на каждом слагаемом $A_{i}, i=1, \ldots, n$, с произвольным элементом $\eta \in C(E(G \oplus H))$ следует, что $\eta \in R_{i} \cong E\left(A_{i}\right), R_{i} \subset \mathbb{Q}$. Поэтому центр кольца $E(G \oplus H)$ изоморфен подкольцу $E$ кольца $E(H)$, причем $P(E,+)=P(G)$.

Пусть $G$ - сепарабельная группа без кручения и $\Omega(G)$ - множество типов всех прямых слагаемых ранга 1 группы $G$. Введем следующее отношение эквивалентности на множестве $\Omega(G)$. Два типа $s, t \in \Omega(G)$ считаем эквивалентными, если существуют $r_{1}, \ldots, r_{n} \in$ $\Omega(G)$ такие, что типы $r_{i}$ и $r_{i+1}$ сравнимы для всех $i=0,1, \ldots, n$, где мы полагаем $r_{0}=s$, $r_{n+1}=t$. Запишем разбиение множества $\Omega(G)$ на непересекающиеся классы эквивалентности: $\Omega(G)=\bigcup_{k \in K} \Omega(k)$. Множество типов $\Omega(G)$ назовем связанным, если $|K|=1$.

Теорема 5. Любые AB-подклассы $\mathbf{F}_{\mathrm{cd}}(A B)$ класса $\mathbf{F}_{\mathrm{cd}} u \mathbf{S}(A B)$ класса $\mathbf{S}$ являются NC-классами.

ДоказАтельство. Пусть $G \in \mathbf{F}_{\mathrm{cd}}(A B)(G \in \mathbf{S}(A B)), \Omega(G)$ - множество всех различных типов прямых слагаемых ранга 1 группы $G$. Если хотя бы одно из прямых слагаемых ранга 1 группы $G$ не почти делимо, то согласно [3], [4] можно построить группу $H$, не изоморфную группе $G$ и такую, что $E(G) \cong E(H)$, причем $r(H)=r(G)$. Пусть теперь все слагаемые ранга 1 группы $G$ почти делимы. Предположим, что $\Omega(G)$ - связанное множество. Покажем, что тогда существует группа $H \in \mathbf{F}_{\mathrm{cd}}(A B)(H \in \mathbf{S}(A B))$ такая, что $G$ не изоморфна $H$ и $C(E(G)) \cong C(E(H))$. Ясно, что $C(E(G)) \cong R \subseteq \mathbb{Q}$. Группу $H$ построим следующим образом: если $r(G)=\infty$, то $H=(R,+)$, если $r(G)=n<\infty$, то $H=\bigoplus_{i=1}^{n+1}(R,+)$. Ясно, что $G$ не изоморфна $H$ и $C(E(G)) \cong C(E(H))$.

Пусть теперь $G=\bigoplus_{i \in I} G_{i}$ - вполне разложимая абелева группа без кручения, и пусть $G=\bigoplus_{\tau \in \Omega(G)} G^{(\tau)}$ - ее каноническое разложение, где

$$
G^{(\tau)}=\bigoplus_{i \in I(\tau)} G_{i}, \quad I(\tau)=\left\{i \in I \mid \tau\left(G_{i}\right)=\tau\right\},
$$

и $\Omega(G)$ - несвязанное множество. Согласно [3] можно считать, что все прямые слагаемые ранга 1 почти делимы и, следовательно, существуют максимальные элементы в $\Omega(G)$. Пусть $\Omega(G)=\bigcup_{k \in K} \Omega(k)$, тогда

$$
G=\bigoplus_{k \in K} G_{k}, \quad \text { где } \quad G_{k}=\bigoplus_{\tau \in \Omega(k)} G^{(\tau)}, \quad \Omega(k)=\Omega\left(G_{k}\right)
$$


и слагаемые $G_{k}, k \in K$, вполне характеристичны в $G$. Поэтому $C(E(G)) \cong \prod_{k \in K} C\left(E\left(G_{k}\right)\right)$ и $C\left(E\left(G_{k}\right)\right) \cong R_{k} \subseteq \mathbb{Q}$ для всех $k \in K$. Для каждой группы $G_{k}, k \in K$, можно построить (см. случай связанного множества $\Omega(G)$ ) неизоморфную группу $H_{k}, k \in K$, такую, что $C\left(E\left(G_{k}\right)\right) \cong C\left(E\left(H_{k}\right)\right)$. Зафиксируем одну из групп $H_{m}, m \in K$, и рассмотрим центр $C\left(E\left(G_{m}\right)\right) \cong C\left(E\left(H_{m}\right)\right)$. Если в группе $G$ существует прямое слагаемое ранга 1 , изоморфное $\left(R_{m},+\right)$, то строим группу $H$ следующим образом:

$$
H=H_{m} \oplus \bigoplus_{m \neq k \in K} G_{k} .
$$

Очевидно, что $C(E(G)) \cong C(E(H))$ и $G \supsetneqq H$.

Пусть в группе $G$ не существует прямого слагаемого ранга 1 , изоморфного $\left(R_{m},+\right)$, и пусть $\tau^{\prime}$ - максимальный элемент множества $\Omega\left(G_{m}\right)$ и $G^{\prime}$ - группа без кручения ранга 1 типа $\tau^{\prime}$. Если ранг $r\left(G^{\left(\tau^{\prime}\right)}\right)$ конечен, то полагаем $H=G \oplus G^{\prime}$. В противном случае полагаем

$$
H=G^{\prime} \oplus \bigoplus_{\tau^{\prime} \neq \tau \in \Omega(G)} G^{(\tau)} .
$$

Каждый раз получаем группу $H$, неизоморфную группе $G$, и $C(E(G)) \cong C(E(H))$.

Пусть теперь $G \in \mathbf{S}(A B)$. Согласно [4] можно считать, что все прямые слагаемые ранга 1 почти делимы и, следовательно, в $\Omega(G)=\bigcup_{k \in K} \Omega(k)$ существуют максимальные элементы. Случай $|K|=1$ рассмотрен выше. Зафиксируем один из максимальных элементов $\tau^{\prime}$ множества $\Omega(G)$. Пусть $G^{\prime}$ - группа без кручения ранга 1 типа $\tau^{\prime}$. Предположим, что в прямом разложении $G$ не существует прямого слагаемого, изоморфного $G^{\prime} \oplus G^{\prime}$. Тогда полагаем $H=G \oplus G^{\prime}$. Пусть теперь в прямом разложении группы $G$ найдется слагаемое, изоморфное $G^{\prime} \oplus G^{\prime}$. Рассмотрим подгруппу $G\left(\tau^{\prime}\right)$ группы $G$. Группа $G\left(\tau^{\prime}\right)$ однородна и вполне характеристична и поэтому сепарабельна. Полагаем $H=G \oplus G\left(\tau^{\prime}\right)$, если $G\left(\tau^{\prime}\right)$ не выделяется прямым слагаемым в $G$, и $H=G \oplus G^{\prime}$, если $G\left(\tau^{\prime}\right)$ выделяется прямым слагаемым в $G$. В каждом из случаев получаем $C(E(G)) \cong C(E(H))$ и $G \supsetneqq H$.

Из работ [3], [4] следует

ПредЛожение 6. Группа А принадлежит классу $\mathbf{F}_{n}(\mathrm{cen})$, если она почти делима и все типы прямых слагаемых ранга 1 фиксированного разложения попарно несравнимы, либо она делимая группа.

СлеДСтвиЕ 7. Класс $\mathbf{F}_{1}$ (cen) состоит в точности из почти делимых групп ранга 1.

\section{СПИСОК ЦИТИРОВАННОЙ ЛИТЕРАТУРЫ}

[1] Л. Фукс, Бесконечные абелевы группы, т. 1, Мир, М., 1974. [2] Л. Фукс, Бесконечные абелевы группы, т. 2, Мир, М., 1977. [3] А. М. Себельдин, Матем. заметки, 11:4 (1972), 403-408. [4] S. Bazzoni, C. Metelli, Conf. Abelian Groups and Their Relationship to the Theory of Modules (INDAM, Rome, 1977), Symp. Math., 23, Academic Press, London-New York, 1979, 259-285.

\section{А. М. Себельдин}

Поступило

Нижегородский государственный

25.10.2006

педагогический университет

E-mail: sebeldinam@rambler.ru

Исправленный вариант

\section{Д. С. Чистяков}

Нижегородский государственный педагогический университет

E-mail: chistyakovds@yandex.ru 